\title{
The long term stability of multilayer walling structures
}

\author{
Elena Korol $^{1}$, Mikhail Berlinov ${ }^{1}$, and Marina Berlinova ${ }^{1, *}$ \\ ${ }^{1}$ Moscow State University of Civil Engineering, Yaroslavskoe shosse, 26, Moscow, 129337, Russia
}

\begin{abstract}
An approach to the assessment of long-term strength and durability of concrete in multi-layer section of enclosing structures by numerical integration of the equations of physics that for practical calculations provides more accurate evaluation of the actual stress-strain state of a bended three-layer element, taking into account the characteristics of deformation layers.
\end{abstract}

\section{Introduction}

Modern construction industry is inconceivable without full assembly construction. With this technology in many parts of the world has realized the possibility of a significant reduction in terms of the construction of various buildings on site. Currently prefabrication housing construction is experiencing a rebirth.

In order to meet modern requirements for heat saving, indoor comfort, architectural expressiveness of buildings and the like, are introduced technology based including on new construction materials. At present, we developed and implemented a variety of approaches to the calculation of constructions made of reinforced concretex [1-5]. For example, considering the influence of the conditions set concrete strength [6] and the mode of loading of concrete structures to level of stress and strain. Calculation of reinforced concrete sandwich elements with the middle layer of concrete with low strength by the deformation model taking into account the full chart of materials allows the state with one voice predict the durability of such structures [7].

Features concrete multilayer structures, along with high-strength concrete was allowed to use not only the concrete of lower strength, but significantly reduce steel consumption.

Prolonged application load and its value is greatly affected by a number of important properties of concrete in multi-layer structures, in particular the strength and deformability. The most common types of loads and sources of stress are factors like power, and nonviolent character: shrinkage and creep of layers; the loads caused during transportation and installation; constant operating load; foundation settlement; wind loads; vibrations and temperature difference between the concrete layers [8,9]. The monolithic cement matrix connecting layers in multilayer panels of different strength concretes provides transfer loads from the outer to the inner layer. As a general rule, as flexible connections in the sandwich panels using connecting members having the required corrosion resistance.

*Corresponding author: marina.tvor@mail.ru 
We know that the relationship between stress and deformation of concrete is a function of time. A gradual increase in deformations over time due to creep property, which can be determined by the amount of rise of deformations at a constant load. In some cases, creep is several times higher than the deformation of the load.

S.V.Aleksandrovsky [10] suggested that the creep is associated mainly with the redistribution of adsorption and capillary-bound water (contained in the newly formed cement stone) depending on the stresses in his body under the influence of an external load. Reducing the water content in a given area of the stone causes shrinkage, and an increase in other parts of the body - the swelling is expressed as, ultimately, to creep deformation.

\section{Results section}

According to the classical theory of creep, an equation of state of concrete is:

$$
\varepsilon_{(t)}=\frac{\sigma_{b}(t)}{E_{b}(t)}+\int_{0}^{t} \frac{\sigma_{b}(\tau)}{E_{b}(\tau)} \theta(t, \tau) d \tau+\int_{0}^{\eta_{\max } t} \mathcal{F}\left(\eta R_{b}(t, \tau)\right) \cdot \phi(\tau) \cdot d \eta,
$$

where: $t$ - time;

$\eta_{\max } t$ - the maximum level of stress on the interval $(0, t)$;

$\varepsilon_{(t)}$ - search deformation;

$\tau$ - the current time;

$\sigma_{b}(\mathrm{t})$ - stress at time $\mathrm{t}$;

$E_{b}(\tau)$ - modulus of elasticity of concrete;

$\sigma_{b}(\tau)$ - current value of stress;

$E_{b}(\tau)$ - current value of the modulus of elasticity;

$\theta(\mathrm{t}, \tau)$ - hereditary function expressing the influence of elastic deformation on a full deformation (refers to the linear part of deformation, for whom the hypothesis about adding strains is adopted);

$\phi(\tau)$ - non-linear hereditory function expressing the effect of stress level (refers to the nonlinear part deformation creep);

$\mathcal{F}\left(\eta R_{b}(t, \tau)\right)$ - is a function of the level of stress;

$\eta=\frac{\sigma_{b}(t)}{R_{b}(t)}$ - stress level;

$R_{b}(t)$-strength of concrete.

Then the function nonlinearity, depending on the level of loading, you can write:

$$
\mathcal{F}\left(\eta \mathrm{R}_{b}(\mathrm{t}, \tau)\right)=\frac{\partial}{\partial \eta} \theta(t, \tau) \frac{\eta}{1-S(t, \tau) \cdot \eta} .
$$

Deformation of creep of concrete $S(t, \tau)$ depends on the duration of loading and does $(t-\tau)$ not depend on the age of the concrete $\tau$.

Change the strength of the concrete in time is:

$$
R_{b}(t)=R_{b}\left[1-\frac{\widehat{m}+1}{N(t)} \int_{0}^{t}\left(\frac{\sigma_{b}(\tau)}{R_{b}}\right)^{\widehat{m}}\right]
$$

Considerable simplification is achieved by using fictitious stress distributions (fig. 1), which is linked to plot deformation relations of the linear theory of creep. 


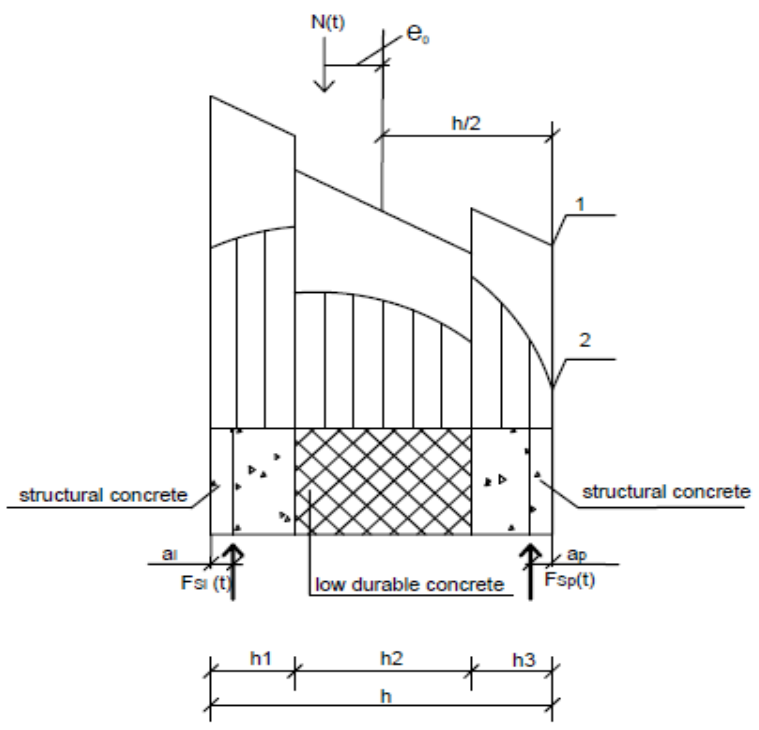

Fig. 1. Stress-strain State of three-layered element: 1 - fictitious plot stresses $\sigma_{\mathrm{f}}(\mathrm{t}), 2$-the actual plot of stress $\sigma_{b}(t)$.

Fictitious stress distributions link with nonlinear plot stress is expressed by the formula:

$$
\sigma_{b}(\tau)=\mathrm{R}_{b}(\mathrm{t})\left[1-\left(1-\frac{x}{x_{R}(t)}\right)^{m(t)}\right]
$$

where $m(t)$ - nonlinearity coefficient of stress distributions:

$$
m(t)=\frac{\frac{m(t)_{o}}{E_{b}}+C_{l}(t, \tau)+C_{n}(t, \tau)}{\frac{1}{E_{b}}+C_{l}(t, \tau)},
$$

$m(t)_{o}$ - parameter $m(t)$ for short-term loading;

$E_{b}$ - modulus of elasticity of concrete;

$C_{l}, C_{n}-$ linear and non-linear measure creep.

Thus, by using nonlinearity coefficient linear relationship between fictitious and non-linear stress plot

$$
\sigma_{f}(t)=m(t) \cdot \sigma_{b}(t)
$$

Using stress distributions, define integral power factors operating in the section of the longitudinal force $\mathrm{N}(\mathrm{t})$ and the bending moment $\mathrm{M}(\mathrm{t})$ :

$$
\begin{aligned}
& N(t)=b \int_{0}^{x(t)} R_{b}(t)\left[1-\left(1-\frac{x}{x_{R}(t)}\right)^{m(t)}\right] d x, \\
& M(t)=b \int_{0}^{x(t)} R_{b}(t) \cdot x\left[1-\left(1-\frac{x}{x_{R}(t)}\right)^{m(t)}\right] d x,
\end{aligned}
$$

where $b$ - is the width of a section, $x$ - is the current coordinate.

Integrating and introducing symbols:

$$
Y_{1}(t)=\left[1-\frac{x(t)}{x_{R}(t)}\right]^{m(t)+1}-1,
$$




$$
Y_{2}(t)=\left[1-\frac{x(t)}{x_{R}(t)}\right]^{m(t)+2}-1
$$

Write an expression for $N(t)$ and $M(t)$ :

$$
\begin{gathered}
N(t)=b R_{b}(t)\left[x(t)+\frac{x_{R}(t) \cdot Y_{1}(t)}{m(t)+1}\right. \\
M(t)=b R_{b}(t)\left[\frac{1}{2} x^{2}(t)-\frac{x_{R}^{2}(t) \cdot Y_{2}(t)}{m(t)+2}+\frac{x_{R}^{2}(t) \cdot Y_{1}(t)}{m(t)+1}\right] .
\end{gathered}
$$

Replacing the ratio $\frac{x(t)}{x_{R}(t)}$ attitudes $\frac{\sigma_{f}(t)}{m(t) R_{b}(t)}$, introducing the simplifying, we obtain an expression for the $\sigma_{b}(t)$ as follows:

$$
\sigma_{b}(t)=R_{b}(t)\left[1+\left(\frac{1,4 R_{b}(t)}{R_{b}}-0,9\right) \cdot\left(1-\frac{\sigma_{\mathrm{f}}(t)}{m(t) \cdot R_{b}(t)}\right) .\right.
$$

The system of equations for the three layered elements:

$$
\left\{\begin{array}{c}
N=F_{b 1}+F_{b 2}+F_{b 3}+F_{s l}+F_{s p} \\
N=M_{b 1}+M_{b 2}-M_{b 3}+F_{s l}\left(x-a_{l}\right)-F_{s p}\left(h-x-a_{p}\right)
\end{array} .\right.
$$

Here are the - components of the longitudinal force:

$F_{b 1}=b \int_{x-h_{1}}^{x} \sigma_{b} d x$ - from the layer of structural concrete in the left brink section;

$F_{b 2}=b \int_{-h-x}^{x-h_{1}-h_{2}} \sigma_{b} d x$ - from the layer of structural concrete right brink section;

$F_{b 3}=b \int_{x-h_{1}-h_{2}}^{x-h_{1}} \sigma_{b 3} d x$ - middle layer of low durable concrete;

- components of the bending moment:

$M_{b 1}=b \int_{x-h_{1}}^{x} x \sigma_{b 1} d x$ - from the layer of structural concrete on left brink;

$M_{b 2}=b \int_{-(h-x)}^{x-h_{1}-h_{2}} x \sigma_{b 2} d x$ - from the structural layer of concrete on the right brink;

$M_{b 3}=b \int_{x-h_{1}-h_{2}}^{x-h_{1}} x \sigma_{b 3} d x-$ from the middle layer of the low durable concrete.

The problem is solved by method of displacement with consideration of the variable rigidity section. At each step of the iterative process, the value of strain on the faces of the panel are made so as to satisfy the conditions of equilibrium.

Then, found $\varepsilon_{l}$ and $\varepsilon_{\mathrm{p}}$ calculated curvature and height of the compressed zone:

$$
\frac{1}{\rho}=\frac{\varepsilon_{l}-\varepsilon_{p}}{h} ; \quad X=\frac{\varepsilon_{l}}{1 / \rho} .
$$

In a manner discussed above, we determine the module of deformation.

Efforts in reinforcement are:

$$
\begin{aligned}
& N_{s l}=A_{s l} \cdot E_{s} \cdot \varepsilon_{s 1}=A_{s l} \cdot E_{s}\left(\varepsilon_{1}-\frac{1}{\rho} a_{\mathrm{l}}\right) \leq A_{s l} \cdot \sigma_{T} \\
& N_{s p}=A_{s p} \cdot E_{s} \cdot \varepsilon_{s p}=A_{s p} \cdot E_{s}\left(\varepsilon_{p}-\frac{1}{\rho}\left(h-a_{p}\right)\right) \leq A_{s p} \cdot \sigma_{T} .
\end{aligned}
$$

With this approach, the deflection is calculated as

$$
f(t)=\frac{1}{\rho}(t) \cdot \frac{l^{2}}{B}
$$

And then you can find the value of the longitudinal force $\overline{\mathrm{N}} \quad$, moment $\overline{\mathrm{M}}$ initial eccentricity e, corresponding to the accepted boundary deformations $\varepsilon_{1}, \varepsilon_{\mathrm{p}}$ and compare $\overline{\mathrm{N}}$ and $\overline{\mathrm{e}}_{0}$ with specified external values $\mathrm{N}$ and $\mathrm{e}_{0}$. 
Here:

$$
\bar{M}=\bar{N}\left(x-\frac{h}{2}+\bar{e}_{0}+f\right), \bar{e}_{0}=\frac{M}{N}-x-f+\frac{h}{2} .
$$

By breaking down the column section in layers, according to Figure 1, repeat the numerical process for each layer. Then, for received values $\varepsilon_{1}$ and $\varepsilon_{\mathrm{p}}$ can be found the modulus of deformation for fibers coincident with edges of element $E_{11}(t)$ and $E_{1 p}(t)$ and respectively calculate the edge fictitious stresses:

$$
\sigma_{f l}=\varepsilon_{l} \cdot E_{l l} u \sigma_{f p}=\varepsilon_{p} \cdot E_{l p} .
$$

From here you can consistently find:

$$
\begin{gathered}
\frac{1}{\rho}=\frac{\varepsilon_{l}-\varepsilon_{p}}{h} ; \quad \varepsilon_{i}=\varepsilon_{l}-\frac{1}{\rho} \cdot \frac{h}{n-1}(i-1) ; f=\frac{1}{\rho} \cdot \frac{l^{2}}{B} \cdot \psi_{\delta} \cdot \varphi_{\delta} ; r=\frac{\sigma_{f l}-\sigma_{f p}}{h} ; \\
\sigma_{\mathrm{f} i}=\sigma_{\mathrm{fl}}-r \cdot \frac{h}{n-1}(i-1) .
\end{gathered}
$$

Here, when $\sigma_{\mathrm{f} i} \leq 0, \sigma_{i}=0$,

when $\sigma_{\mathrm{f} i} \leq m_{i} R_{i} \sigma_{i}=R_{i}\left[1-\left(1-\frac{\sigma_{\mathrm{fi}}}{m_{i} R_{i}}\right)^{m_{i}}\right]$,

when $\sigma_{\mathrm{f} i}>m_{i} R_{i} \sigma_{i}=R_{i}\left[1+\left(\frac{1.4 R_{i}}{R_{b}}-0.9\right) \cdot\left(1-\frac{\sigma_{\mathrm{fi}}}{m_{i} R_{i}}\right)\right]$.

It is convenient to lead the fiber located in the middle section, and here modulus for each i-th fibers will be equal,

$$
E_{i}=\frac{\sigma_{i}}{\varepsilon_{i}}
$$

For fiber, which is located in the middle section $\mathrm{Z}$ all reduction coefficients for the reinforcement and concrete are

$$
n_{\mathrm{sl}}=\frac{E_{s}}{\mathrm{Z}}, \quad n_{\mathrm{sp}}=\frac{E_{s}}{\mathrm{Z} \cdot \Psi_{s}}, \quad n_{i}=\frac{E_{i}}{Z},
$$

Thus, the reduction area of such section

$$
A=n_{s l} \cdot A_{s l}+n_{s p} \cdot A_{s p}+b \sum_{i=1}^{n-1} n_{i} \frac{h}{n-1} .
$$

The static moment with respect to the left side

$$
S=n_{s l} \cdot A_{s l} \cdot a_{l}+n_{s p} \cdot A_{s p} \cdot\left(h-a_{p}\right)+b \sum_{i=1}^{n-1} n_{i}\left(\frac{h}{n-1}\right)^{2}(i-0,5)
$$

The distance from the left side to the center of gravity of the section:

$$
y=\frac{S}{A}
$$

and the moment of inertia of the cross section shown will be equal to:

$$
\begin{gathered}
Y=n_{s l} \cdot A_{s l}\left(y-a_{l}\right)^{2}+n_{s p} \cdot A_{s p} \cdot\left(h-y-a_{p}\right)^{2}+b \sum_{i=1}^{n-1}\left[\frac{n_{i}\left(\frac{h}{n-1}\right)^{3}}{12}+n_{i} \frac{h}{n-1}(y-\right. \\
\left.\left.\frac{h}{n-1}(i-0,5)\right)^{2}\right]
\end{gathered}
$$

Hence, the relevant moments of resistance: 


$$
W_{\mathrm{l}}=\frac{Y}{y} ; \quad W_{\mathrm{p}}=\frac{Y}{h-y} .
$$

Thus, when taken $\varepsilon_{1}$ and $\varepsilon_{\mathrm{p}}$ can be found:

$$
\begin{gathered}
\bar{\sigma}_{f l}=N\left[\frac{1}{A}+\frac{1}{W_{l}}\left(y-\frac{h}{2}+e_{0}+f\right)\right. \\
\bar{\sigma}_{f p}=N\left[\frac{1}{A}-\frac{1}{W_{p}}\left(y-\frac{h}{2}+e_{0}+f\right)\right], \\
\varepsilon_{l}=\frac{\bar{\sigma}_{f l}}{Z}, \varepsilon_{p}=\frac{\bar{\sigma}_{f p}}{Z} .
\end{gathered}
$$

On condition

$\left|\varepsilon_{1}-\bar{\varepsilon}_{1}\right|<0.05 \%$ и $\left|\varepsilon_{\mathrm{p}}-\bar{\varepsilon}_{\mathrm{p}}\right|<0.05 \%$ о

you can proceed to the next load or moment of time, otherwise the value adopted for the deformations of the next iteration $\mathrm{p}$ can be calculated as

$\varepsilon_{1}=\frac{\varepsilon_{1}+\bar{\varepsilon}_{1}}{2}$ и $\varepsilon_{\mathrm{p}}=\frac{\varepsilon_{\mathrm{p}}+\bar{\varepsilon}_{\mathrm{p}}}{2}$.

And then you need to repeat the calculation until convergence.

\section{Conclusions}

A feature of the proposed method of calculation of the practical strength of multi-layer walls of concrete of different strength using physical dependency is a representation of nonlinear creep deformation as the sum of the strain and stress levels in time. The presented method will improve the structural safety, and to improve energy efficiency of such constractions in public buildings for temporary and permanent stay of people. The solution of this problem offers the prospect of developing the theoretical foundations of forecasting multilayer walling strength at any stage of their operation.

\section{References}

1. M.V. Berlinov, E.A. Makarenkov, Bulletin MGSU, 11, 26 (2013)

2. M.N. Berlinova, A.V. Tvorogov. Natural and engineering sciences. 6 (84), 530 (2015)

3. E.M. Zveryaev, G.I.Makarov. Mechanical engineering and construction of structures and facilities. 4, 6 (2012)

4. N.V. Klyueva. Concrete and reinforced concrete. 3, 22 (2008)

5. A.N. Davidyuk, A.A. Davidyuk, Concrete and reinforced concrete, 10, 1 (2008)

6. E. M. Zverjaev, M. V. Berlinov, M. N. Berlinova. Int. J. of Applied Engineering Research. 11(8) 5811 (2016)

7. E. Korol, P.Kagan, T.Barabanova. AMM. 738, 444 (2015)

8. E. A. Korol, M. V. Berlinov, M. N. Berlinova. Procedia Engineering, 292 (2016)

9. E.A. Korol, Yu.A. Kharkin, Book of Reports of XX Russian-Polish-Slovak Workshop “Theoretical Fundamentals of Construction". 401 (2011)

10. S. V. Aleksandrovsky. Durability of external walling (2004) 